\title{
Macroscopic quantum tunneling in a de SQUID: Instanton splitting
}

\author{
C. Morais Smith \\ Theoretische Physik, Eidgenössische Technische Hochschule-Hönggerberg, CH-8093 Zürich, Switzerland \\ and Universidade Estadual Paulista Julio de Mesquita, UNESP, 17100 Bauru, Brazil \\ B. Ivlev \\ Theoretische Physik, Eidgenössische Technische Hochschule-Hönggerberg, CH-8093 Zürich, Switzerland \\ and L. D. Landau Institute for Theoretical Physics, 1179940 Moscow, Russia

\section{G. Blatter} \\ Theoretische Physik, Eidgenössische Technische Hochschule-Hönggerberg, CH-8093 Zürich, Switzerland
}

(Received 24 May 1993; revised manuscript received 22 October 1993)

\begin{abstract}
The theory of macroscopic quantum tunneling is applied to a current-biased dc SQUID which constitutes a system of two interacting quantum degrees of freedom coupled to the environment. The decay probability is obtained in the exponential approximation for the overdamped case. Close to the critical driving force of the system, the decay of the metastable state is determined by a unique instanton solution describing the symmetric decay of the phases in each of the two Josephson juctions. Upon reducing the external driving force a new regime is reached where the instanton splits. The doubling of the decay channels reduces the decreasing of the decay rate in the quantum regime. A current-temperature phase diagram is constructed based on the Landau theory of phase transitions. Depending on the external parameters the system develops either a first- or a second-order transition to the split-instanton regime.
\end{abstract}

\section{INTRODUCTION}

During the last decade the subject of macroscopic quantum phenomena has attracted a great deal of interest. Macroscopic systems are inherently coupled to their environment which makes it often necessary to take dissipation into account. ${ }^{1-5}$ Some scientifically interesting as well as technologically important examples exhibiting this kind of phenomena are superconducting quantum interference devices (SQUID) and current-biased Josephson junctions (JJ). In the former, the macroscopic variable is the flux trapped in the ring, while in a $\mathrm{JJ}$, the phase of the junction is the variable which can be subject to quantum effects. In both examples, the decay probability is determined in the exponential approximation by the action evaluated for the (classical) extremal trajectory in an imaginary time formulation (instanton). ${ }^{6-8}$

In this work we are interested in the quantum dynamics of a system which is equivalent to an interacting twoparticle problem, i.e., the decay of a metastable state in a dc SQUID. The device contains two JJ which makes it a particularly interesting model system as it goes beyond a single degree of freedom. Under well-defined conditions, it develops an instability where the action becomes extremal along two close-lying trajectories. Such a splitting of the instanton makes the two phases decay asymmetrically and strongly influences the value of the decay rate. A deeper understanding of the SQUID behavior is of great technological importance since these devices are used, for instance, as magnetic-flux detectors, allowing the construction of very sensitive magnetometers. ${ }^{9}$

The macroscopic quantum tunneling (MQT) behavior of a dc SQUID with a very small ring inductance or with currents flowing in the two junctions nearly in phase has been considered before by Chen. ${ }^{10}$ In both cases, only the one-instanton solution arises. Ivlev and Ovchinnikov ${ }^{11}$ have calculated the instanton splitting in a dc SQUID for the underdamped case. Here, we concentrate on the effect of dissipation and study the decay process in the overdamped limit.

The standard method to treat the effect of damping on MQT is the theory introduced by Caldeira and Leggett (CL) ${ }^{1,2}$ In their formalism the dissipation is treated by coupling the particle to a thermal bosonic bath. The CL theory assumes that each degree of freedom of the environment is only slightly perturbed by the coupling with the particle such that the bath can be represented by an ensemble of harmonic oscillators. Notice that this weakcoupling hypothesis does not exclude the possibility of strong dissipation, since the environment is composed of an infinite number of degrees of freedom. In the CL model, the tunneling rate for a dissipative macroscopic quantum variable is computed at zero temperature. The formalism has been generalized to finite temperatures by Larkin and Ovchinnikov ${ }^{3}$ in the limit of strong dissipation, and by Grabert, Weiss, and Hänggi ${ }^{4,5}$ in the general case. For a recent review see Refs. 12 and 13.

In the present work we consider the decay mode of a metastable state in a dc SQUID consisting of two identical $\mathbf{J J}$ arranged symmetrically in a superconducting loop and connected to the outside world by two leads (Fig. 1). The device is current biased near to criticality, i.e., the current $I_{i}, i=1,2$, in each branch is chosen to be slightly smaller than the critical current $I_{c}$. The critical current in a $\mathrm{JJ}$ is nothing but the maximum current which can be transported by Cooper pairs. When the current exceeds 


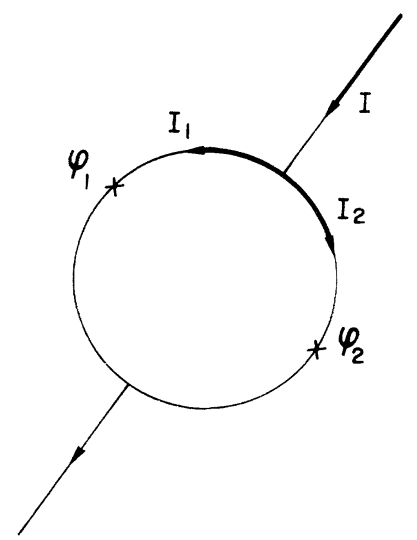

FIG. 1. dc SQUID consisting of two identical Josephson junctions arranged symmetrically in a superconducting loop. The phase differences $\varphi_{1}$ and $\varphi_{2}$ in each $\mathrm{JJ}$ are the macroscopic coordinates decaying out of a metastable state. In order to observe quantum effects, the system is current biased near to criticality, i.e., the currents $I_{1}$ and $I_{2}$ in each branch are slightly smaller than the critical current $I_{c}$. The effective shape of the potential is modified by changing the total current $I=I_{1}+I_{2}$.

this maximum value, a finite voltage appears across the junction, indicating that the additional current has to be carried by quasiparticles.

The system under consideration develops a very interesting behavior: upon changing external parameters such as the bias current $I=I_{1}+I_{2}$ or the temperature $T$, several physically different situations can be realized. At high temperatures and currents, the system is in the classical regime and decays through a unique saddle by thermal activation. Upon reducing the current a new phase arises at $I=I_{c s}$, where the saddle splits into two lower ones and the decay probability of the thermal process increases. Instead, if the current is kept constant and the temperature is reduced, the system enters the quantum regime and different regions can be reached: For very high currents, the coupling between the two JJ is strong and both phases decay symmetrically (singleinstanton region). For lower currents, the coupling becomes weaker and the decay ceases to be symmetric. In this case, two close-lying decay channels become available, i.e., the instanton splits into two. The bifurcation of the trajectory reduces the action and consequently, the decay rate determined by the split-instanton solution is larger than the rate obtained for the single instanton in the same range of temperature and current. The phenomenon of instanton splitting occurs at currents larger than $I_{c s}$, i.e., in the region where the classical regime is still governed by a single saddle. For smaller currents $I<I_{c s}$ the quantum regime naturally involves two instanton trajectories. Identifying the action with a thermodynamic potential, we can reformulate the problem in terms of the Landau theory of phase transitions. On this basis, the transition between the one-instanton and the two-instanton phases can be either continuous (a second-order phase transition), or it can be discontinuous (a first-order phase transition). The order of the phase transition depends on the bias current and on the temperature. For small values of the external current and close to the crossover temperature $T_{0}$ to the classical regime, the system develops a first-order phase transition, while for temperatures close to zero and higher bias current, the transition is continuous.

The outline of this paper is as follows: In Sec. II we derive and discuss the action describing our system. In Sec. III we calculate the saddle points with the corresponding instantons. The decay rate in the exponential approximation is computed in Sec. IV and the phase diagram for the four different current-temperature regimes (classical single saddle, classical double saddle, quantum single instanton, and quantum split instanton) is discussed in detail. The conclusions are presented in Sec. V.

\section{EUCLIDEAN ACTION}

The probability for the decay of a metastable state at finite temperature $T$ is related to the imaginary part of the free energy $F$

$$
\Gamma=\frac{2}{\hbar} \operatorname{Im} F
$$

In the semiclassical approximation, it can be shown that the imaginary part of $F$ is dominated by the classical trajectory in the inverted potential (bounce or instanton) and the above expression takes the form ${ }^{8}$

$$
\Gamma=f e^{-S_{E} / \hbar}
$$

where $S_{E}$ is the Euclidean (imaginary time) action evaluated along the instanton trajectory and $f$ is a prefactor, which is obtained by considering fluctuations around this trajectory. The instanton path is a saddle point of the action, i.e., a path which extremizes the action. The Euclidean action of a system consisting of two identical coupled Josephson junctions in the presence of a bias current is

$$
S_{E}=S_{j}+S_{i}+S_{d}
$$

where

$$
\begin{aligned}
S_{j}=\int_{0}^{\hbar / T} d \tau & \left\{\left(\frac{\Phi_{0}}{2 \pi}\right]^{2} \frac{C}{2}\left[\int \frac{\partial \varphi_{1}}{\partial \tau}\right]^{2}+\left(\frac{\partial \varphi_{2}}{\partial \tau}\right)^{2}\right] \\
& +E_{J}\left[\left(1-\cos \varphi_{1}\right)+\left(1-\cos \varphi_{2}\right)\right] \\
& \left.-E_{J} \frac{I}{2 I_{c}}\left(\varphi_{1}+\varphi_{2}\right)\right\}
\end{aligned}
$$

is the action of two independent $\mathbf{J J}$. Here and in the following we have set the Boltzmann constant equal to unity, $k_{B}=1$. The first term is the kinetic energy, where the capacitance $C$ of the junction plays the role of the "mass" in the system; $\Phi_{0}=h c / 2 e$ is the flux quantum; $\varphi_{1}$ and $\varphi_{2}$ are the phase differences across the two junctions which are periodic functions of the imaginary time $\tau$ with a period $\hbar / T$. The second term is the potential energy of the junctions, $E_{J}=\left(\Phi_{0} / 2 \pi\right) I_{c}$, where $I_{c}$ is the critical current of an individual junction. The last term 
represents the driving force in the system, which can be externally controlled via a change of the bias current $I$.

The interaction between the two $\mathrm{JJ}$ is given by

$$
S_{i}=\int_{0}^{\hbar / T} d \tau \frac{E_{J}^{2}}{2 L I_{c}^{2}}\left(\varphi_{1}-\varphi_{2}\right)^{2}
$$

where $L$ is the total inductance of the loop. The expression (3b) corresponds to the classical energy of a loop with total inductance $L$ and enclosing a flux $\Phi, E=\Phi^{2} / 2 L$. The value of $\Phi$ is determined from the condition that the macroscopic phase of the wave function must be single valued around the loop. In a dc SQUID, the gauge-invariant phase differences $\varphi_{1}$ and $\varphi_{2}$ across the junctions determine the flux $\Phi=\left(\Phi_{0} / 2 \pi\right)\left(\varphi_{1}-\varphi_{2}\right)$. The present system is intermedi- ate between a zero- and a one-dimensional problem as realized in a rf SQUID $(d=0)($ Refs. 1,2$)$ or a tunneling vortex line $(d=1)$ (Refs. 14,15), for example. For the vortex problem, the macroscopic variable is given by the displacement field $u(x, \tau)$, where $x$ denotes the coordinate along the string. The interaction energy $E_{J}^{2}\left(\varphi_{1}-\varphi_{2}\right)^{2} / 2 L I_{c}^{2}$ in the dc SQUID then maps to the elastic energy $\int^{c} d x \varepsilon_{l}\left(\partial_{x} u\right)^{2} / 2$ in the vortex problem with $\varepsilon_{l}$ denoting the line tension of the string.

The last term in the action, $S_{d}$, is the dissipative contribution due to the coupling to the environment. Here we consider the resistively shunted junction (RSJ) model such that we can use the Caldeira-Leggett model in order to describe the coupling between the phases $\varphi_{i}, i=1,2$, of the $\mathrm{JJ}$ and the heat bath;

$$
S_{d}=-\frac{\eta}{2 \pi} \sum_{i=1,2} \int_{0}^{\hbar / T} d \tau\left(\frac{\partial \varphi_{i}}{\partial \tau}\right) \int_{0}^{\hbar / T} d \tau_{1}\left(\frac{\partial \varphi_{i}}{\partial \tau_{1}}\right) \ln \left|\sin \left[\frac{\pi T}{\hbar}\left(\tau-\tau_{1}\right)\right]\right|
$$

with $\eta$ denoting the phenomenological friction coefficient.

The action (3) describes a situation with a vanishing external magnetic flux threading the loop of the SQUID. If the external flux is nonzero but an integral number of flux quanta, the action can still be written in the form (3). For arbitrary external flux, the SQUID acquires an effective asymmetry. However, our results are general and the splitting of the instanton occurs in any case.

The oscillatory cosine term and the driving term proportional to $I / I_{c}$ [both in (3a)] combined with the interaction potential in (3b) give rise to the tilted washboard potential, which is shown in Fig. 2(a). The minima are arranged along the diagonal $\varphi_{1}=\varphi_{2}$ and an illustration is given in Fig. 2(b), where the equipotential lines are plotted. Experimentally, macroscopic quantum tunneling is studied at currents close to criticality, i.e., $2 I_{c}-I \ll I_{c}$, when the effective potential barrier becomes small. In this limit, the potential energy can be approximated by a cubic form. Introducing the dimensionless variables,

$$
p=\frac{\varphi_{1}+\varphi_{2}}{3 \varepsilon}+\frac{\pi}{\varepsilon}+\frac{2}{3} \text { and } q=\frac{\varphi_{1}-\varphi_{2}}{3 \varepsilon},
$$

the Euclidean action can be rewritten in the form (neglecting a constant term),

$$
\begin{aligned}
S_{E}=g \int_{-\pi}^{\pi} d z & \left\{\frac{G}{2}\left(\frac{\partial p}{\partial z}\right)^{2}+\frac{G}{2}\left[\frac{\partial q}{\partial z}\right)^{2}+V(p, q)\right. \\
& -\frac{T}{\pi T_{0}} \frac{\partial p}{\partial z} \int_{-\pi}^{\pi} d z_{1} \frac{\partial p}{\partial z_{1}} \ln \left|\sin \left(\frac{z-z_{1}}{2}\right)\right| \\
& \left.-\frac{T}{\pi T_{0}} \frac{\partial q}{\partial z} \int_{-\pi}^{\pi} d z_{1} \frac{\partial q}{\partial z_{1}} \ln \left|\sin \left(\frac{z-z_{1}}{2}\right)\right|\right\},
\end{aligned}
$$

where the effective potential is

$$
V(p, q)=\left(p^{2}-\frac{1}{2} p^{3}\right)+q^{2}(1+\alpha)-\frac{3}{2} p q^{2} .
$$

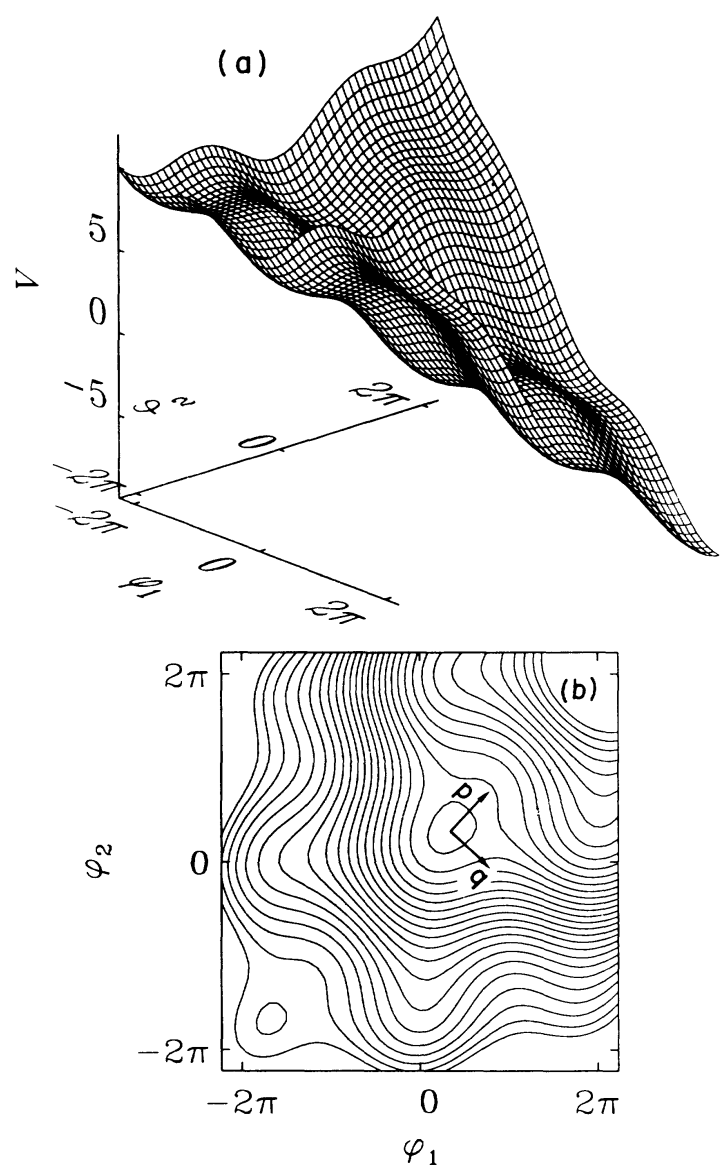

FIG. 2. (a) Tilted washboard potential acting on the phase differences $\varphi_{1}$ and $\varphi_{2}$ in the presence of a bias current $I$. The shape of the potential results from a periodic cosine term (potential energy of the junctions) combined with the bias driving term $\left[\propto I\left(\varphi_{1}+\varphi_{2}\right)\right]$ and with the interaction term $\left[\propto\left(\varphi_{1}-\varphi_{2}\right)^{2} / L\right]$. (b) Equipotential lines plotted for the tilted washboard potential given in (a). The minima are located along the diagonal $\varphi_{1}=\varphi_{2}$. Depending on the external parameter $I$, the phases $\varphi_{1}$ and $\varphi_{2}$ decay from the metastable state following the diagonal (single-instanton phase) or following two closelying trajectories with $q \neq 0$ (split-instanton phase). 
Here we have introduced the dimensionless mass $G=8 \pi^{2} C E_{J} T^{2} / \varepsilon I_{c}^{2} \hbar^{2}$, the dimensionless time $z=2 \pi T \tau / \hbar$, the unit of action $g=9 \hbar E_{J} \varepsilon^{3} / 8 \pi T$, and the crossover temperature $T_{0}=\hbar \varepsilon E_{J} / 2 \pi \eta$, as well as the important driving parameter,

$\alpha=\frac{2}{\beta \varepsilon} \quad$ with $\varepsilon=\sqrt{\left(2 I_{c}-I\right) / I_{c}}$ and $\beta=\left(\frac{2 \pi}{\Phi_{0}}\right) L I_{c}$.

The parameter $g$ is supposed to be large enough to allow us to treat the problem in the semiclassical approxi-
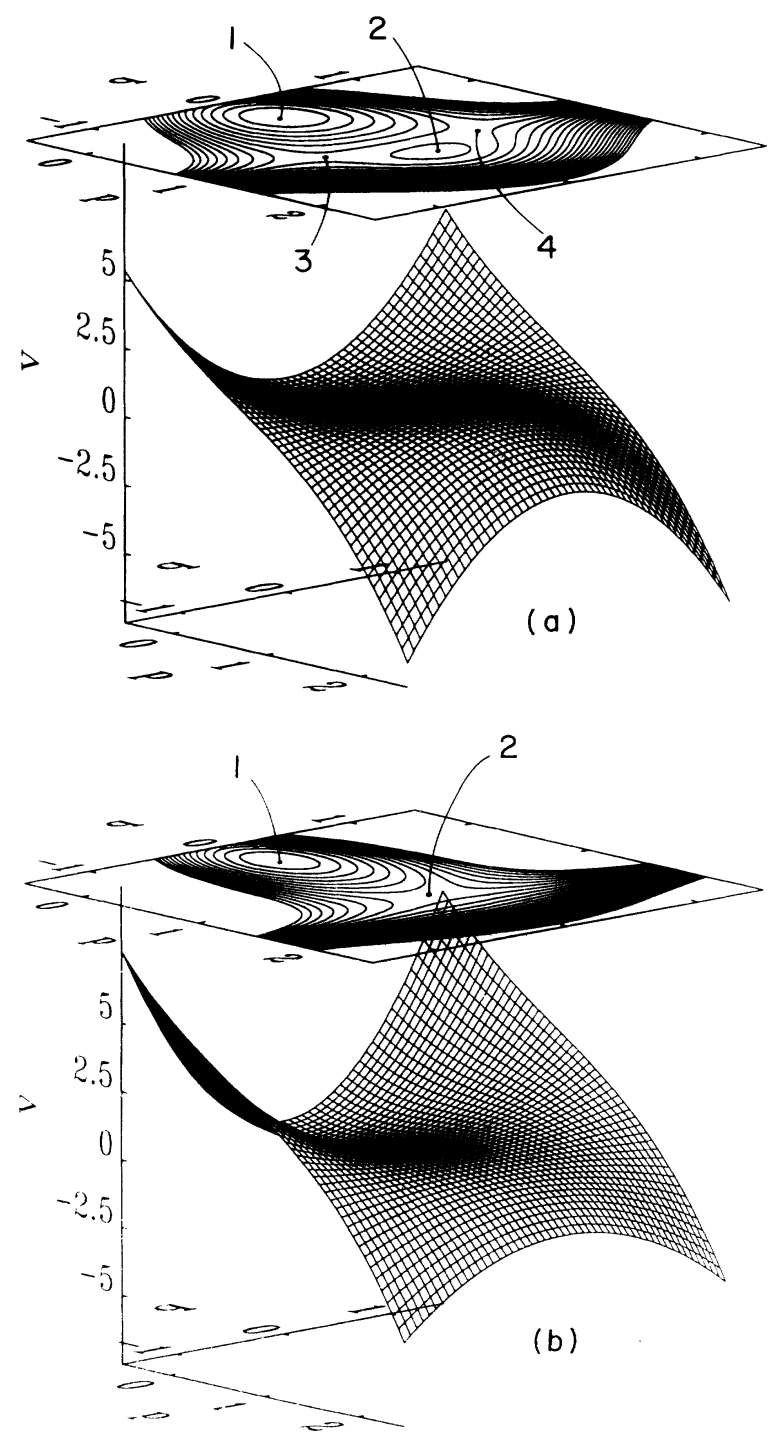

FIG. 3. Detailed view of the region close to a metastable minimum of the effective potential expressed in terms of the dimensionless variables $p$ (center of mass) and $q$ (relative coordinate). Choosing the bias current $I$ close to its critical value, $2 I_{c}-I<<I_{c}$, the potential can be approximated by a cubic form. The shape of the potential is controlled by the driving parameter $\alpha$. (a) At small values of currents $I(\alpha<1)$ the potential has a minimum at $\left(p_{1}, q_{1}\right)$, a maximum at $\left(p_{2}, q_{2}\right)$, and two saddle points, $\left(p_{3}, q_{3}\right)$ and $\left(p_{4}, q_{4}\right)$. (b) For a current $I$ corresponding to the value $\alpha \geq 1$, the points 3 and 4 merge into 2 , which becomes the new saddle point. mation, $g / \hbar>>1$, and small enough to lower the height of the barrier in order that quantum tunneling can be observed (note that $\varepsilon$ is a small parameter, since we are considering bias currents $I$ close to $2 I_{c}$ ). The parameter $\alpha$ controls the interaction between the phases $\varphi_{1}$ and $\varphi_{2}$. It is important to remark that the limit of small inductance $L$ (small $\beta$ ) (Ref. 10) is different from the limit of small $\varepsilon$ considered in this work. Although the two parameters appear in an equivalent way in the expression for $\alpha$, the parameter $g$ also contains a factor $\varepsilon^{3}$ and hence, the two quantities $L$ and $\varepsilon$ play different roles. The latter can be clearly visualized by inspection of Eqs. (3a) and (3b); in fact, the limit of small inductance $L \rightarrow 0$ emphasizes the term quadratic in the phases, while changing the external current $I$ (and hence $\varepsilon$ ) the term linear in the phases is affected. This allows us to drive the system into a situation where the instanton splits, in contrast to Ref. 10.

Let us now analyze more carefully the effective potential $V(p, q)$. Figure 3 shows its behavior for different values of $\alpha$. For $\alpha<1$ (small currents $I$ ), there is an absolute minimum at $p_{1}=q_{1}=0$. An absolute maximum occurs at $p_{2}=\frac{4}{3}, q_{2}=0$, and two saddle points appear at $p_{3,4}=2(1+\alpha) / 3, q_{3,4}= \pm 2 \sqrt{1-\alpha^{2}} / 3$ [see Fig. 3(a)]. For the critical value $\alpha=1$, the points 3 and 4 merge into the point 2, which becomes the new and unique saddle point in the regime $\alpha>1$ [Fig. 3(b)]. From these static considerations one could expect that for large $\alpha$, a unique (dynamic) instanton solution is developed, whereas for small $\alpha$, two separated solutions with $q(z) \neq 0$ should occur. It turns out that such a type of instanton splitting occurs indeed, however, the critical value of $\alpha$ depends on the temperature $T$ with $\alpha_{c}\left(T_{0}\right)=1$ only at the crossover temperature $T_{0}$ where the dynamics become classical. The exact shape of the critical line $\alpha_{c}(T)$ will be obtained in Sec. III.

\section{SADDLE POINTS AND INSTANTON SPLITTING}

In a next step, we look for paths which extremize the above action. This was done by Ivlev and Ovchinnikov ${ }^{11}$ for the dissipation-free system with $\eta=0$. Here, we are interested in the opposite limit, where the dissipation is very strong, $\eta / \hbar>>1$. In the following we will neglect the kinetic terms in Eq. (4), supposing that they are much smaller than the dissipative ones (overdamped regime). Applying the variational principle to the action, we obtain the classical equations of motion for imaginary times,

$$
-2 p+\frac{3}{2} p^{2}+\frac{3}{2} q^{2}=\frac{T}{\pi T_{0}} \int_{-\pi}^{\pi} d z_{1} \frac{\partial p}{\partial z_{1}} \cot \left(\frac{z-z_{1}}{2}\right),
$$

$$
-2(1+\alpha) q+3 p q=\frac{T}{\pi T_{0}} \int_{-\pi}^{\pi} d z_{1} \frac{\partial q}{\partial z_{1}} \cot \left(\frac{z-z_{1}}{2}\right)
$$

Equation (5b) can be trivially satisfied by setting $q(z)=q_{0}(z)=0$. In this case, the present problem reduces to that of a single Josephson junction, i.e., one degree of freedom $(p)$ interacts with a thermal bath at a 
finite temperature $T$. The solution of the remaining equation (instanton) has been obtained by Larkin and Ovchinnikov. ${ }^{3}$ It is given by

$$
p_{0}(z)=\frac{4}{3}\left[\frac{T}{T_{0}}\right)^{2} \frac{1}{1-\cos z \sqrt{1-\left(T / T_{0}\right)^{2}}},
$$

where

$$
T_{0}=\frac{\hbar E_{J}}{2 \pi \eta} \varepsilon(I)
$$

is the crossover temperature separating the classical from the quantum regime. ${ }^{3}$ Inserting $p_{0}, q_{0}$ back into (4) we obtain the action

$$
S_{0}=4 \pi \eta \varepsilon^{2}(I)\left[1-\frac{1}{3}\left(\frac{T}{T_{0}}\right)^{2}\right] .
$$

Next, we investigate the possibility that the dynamical equations (5a) and (5b) may develop a second solution which further reduces the action (4). In particular, we look for a solution $p, q$ which bifurcates away from the solution $p_{0}, q_{0}$ at some critical value of the dynamical parameter $\alpha$. Defining $p(z)=p_{0}(z)+\widetilde{p}(z) \quad$ and $q(z)=q_{0}(z)+\widetilde{q}(z)$, we can rewrite the action in the compact form,

$$
\begin{aligned}
S= & S_{0}+\frac{g}{2} \int_{-\pi}^{\pi} d z[\widetilde{q} \hat{Q} \widetilde{q}+\widetilde{p} \hat{P} \widetilde{p}] \\
& -\frac{g}{2} \int_{-\pi}^{\pi} d z \widetilde{p}^{3}-\frac{3 g}{2} \int_{-\pi}^{\pi} d z \widetilde{p} \widetilde{q}^{2},
\end{aligned}
$$

where we have introduced the two operators

$$
\begin{aligned}
\hat{\boldsymbol{P}}=\left.\frac{\delta^{2} S}{\delta p^{2}}\right|_{p_{0}, q_{0}}= & 2-3 p_{0}(z) \\
& +\frac{T}{\pi T_{0}} \int_{-\pi}^{\pi} d z_{1} \cot \left(\frac{z-z_{1}}{2}\right) \frac{\partial}{\partial z_{1}}
\end{aligned}
$$

and

$$
\widehat{Q}=\left.\frac{\delta^{2} S}{\delta q^{2}}\right|_{p_{0}, p_{0}}=\hat{P}+2 \alpha .
$$

Making use of the instanton technique developed by Callan and Coleman, ${ }^{6,7}$ we know that the operator $\hat{P}$ does not only have a zero eigenvalue (translation mode), but also a negative one (unstable mode) which is responsible for the imaginary part in the energy and hence, for the finite decay rate of the metastable state. From Eq. (1) we immediately see that the value of the parameter $\alpha$ plays a critical role in this problem. For large $\alpha$, the spectrum of $\hat{Q}$ is positive definite and all the $q$ modes are stable. Upon decreasing $\alpha$ (e.g., via reduction of $I$ ) the lowest eigenvalue of $\hat{Q}$ can become negative and an unstable $q$ mode is developed. The value of $\alpha$ where this instability occurs for the first time is defined as the critical value $\alpha_{c}$. Hence, adding $2 \alpha_{c}$ to the operator $\hat{\boldsymbol{P}}$ shifts the negative eigenvalue to zero. For $\alpha>\alpha_{c}$, only $\hat{P}$ has a negative eigenvalue and the action is extremal for the trajectory $\left[p_{0}(z), q_{0}(z)\right]$. On the other hand, for $\alpha<\alpha_{c}$, the operator $\hat{Q}$ also acquires a negative eigenvalue, $2\left(\alpha-\alpha_{c}\right)<0$, and a "dangerous" mode is developed. As a consequence, the single instanton present for $\alpha>\alpha_{c}$ will split into two instantons at $\alpha<\alpha_{c}$.

In terms of the operators (10), the equations of motion (5a) and (5b) can be written in the equivalent form,

$$
\begin{aligned}
& \hat{P} \widetilde{p}=\frac{3}{2} \widetilde{q}^{2}+\frac{3}{2} \widetilde{p}^{2}, \\
& \hat{Q} \widetilde{q}=3 \widetilde{p} \widetilde{q} .
\end{aligned}
$$

Our task now is to determine the corrections $\widetilde{p}$ and $\widetilde{q}$ for $\alpha<\alpha_{c}$. To lowest order, we can set $\widetilde{p}=0$ and $\widetilde{q}$ is the solution of the (linear) eigenvalue problem,

$$
\hat{Q} \widetilde{q}=0 \text {. }
$$

Solving Eq. (12) we thus immediately obtain the critical parameter $\alpha_{c}$ where the spectrum of $\hat{Q}$ goes to zero, $\widehat{Q}\left(\alpha_{c}\right) q_{1}=0$. Here $q_{1}$ denotes the eigenfunction of $\hat{Q}$ belonging to the lowest eigenvalue $2\left(\alpha-\alpha_{c}\right)$, i.e., $\widehat{Q} q_{1}=2\left(\alpha-\alpha_{c}\right) q_{1}$.

Expanding $\widetilde{q}(z)$ in a Fourier series,

$$
\widetilde{q}(z)=\sum_{n=-\infty}^{\infty} C_{n} e^{i n z},
$$

and remembering that ${ }^{16}$

$$
\cot \left(\frac{z-z_{1}}{2}\right)=2 \sum_{m=1}^{\infty} \sin \left[m\left(z-z_{1}\right)\right],
$$

we obtain the following equation for the Fourier coefficients $C_{n}$,

$$
|n| C_{n}+(1+\alpha) \frac{T_{0}}{T} C_{n}=2 \sum_{m=-\infty}^{\infty} C_{m} e^{-b|m-n|},
$$

where

$$
b \equiv \frac{1}{2} \ln \left[\frac{T_{0}+T}{T_{0}-T}\right) .
$$

Using the ansatz

$$
C_{n}=B e^{-b|n|}(A+|n|)
$$

( $A, B$ constants) it is straightforward to verify that we obtain a solution if the conditions

$$
A=\frac{\alpha T_{0}}{T} \text { and }-\alpha^{2}+\alpha+1-\left(\frac{T}{T_{0}}\right)^{2}=0,
$$

are fulfilled. Since Eq. (15) is linear in $C_{n}$, the overall amplitude $B$ cannot be obtained at this stage, but has to be determined by taking higher-order terms in Eq. (11) into account. Equation (18) defines the critical parameter $\alpha_{c}(T)$ below which the lowest eigenvalue of $\hat{Q}$ becomes negative,

$$
\alpha_{c}=\frac{1}{2}+\sqrt{\frac{5}{4}-\left(T / T_{0}\right)^{2}} .
$$

Note that with the above calculation we have simultaneously determined the eigenfunction $q_{1}(z)$ correspond- 
ing to the negative eigenvalue $2\left(\alpha-\alpha_{c}\right)$ of $\widehat{Q}$ in the region $\alpha<\alpha_{c}$, as well as the lowest order approximation $\widetilde{q}^{(0)}(z)$ to the split-instanton solution $\widetilde{q}(z) \neq 0$ appearing below $\alpha_{c}$,

$$
\begin{aligned}
\widetilde{q}(z) \approx \widetilde{q}^{(0)}(z) & =B q_{1}(z) \\
& =B \sum_{n=-\infty}^{\infty} e^{-b|n|}\left(\frac{\alpha_{c} T_{0}}{T}+|n|\right) e^{i n z} .
\end{aligned}
$$

The condition (18) relates the current $I$ with the temperature $T$ and as such determines the critical temperature $T_{s}(\alpha)$ below which the two instantons $\left(p_{0}, \pm B q_{1}\right)$ occur. As in the classical regime, we can verify that this bifurcation appears in the region $\alpha<\alpha_{c}$, i.e., in the lowcurrent regime. In this region, the symmetric solution $\left[q_{0}(z)=0\right]$ is still valid. However, it can be easily verified that the substitution of the solutions $\pm B q_{1}(z)$ into the ac-

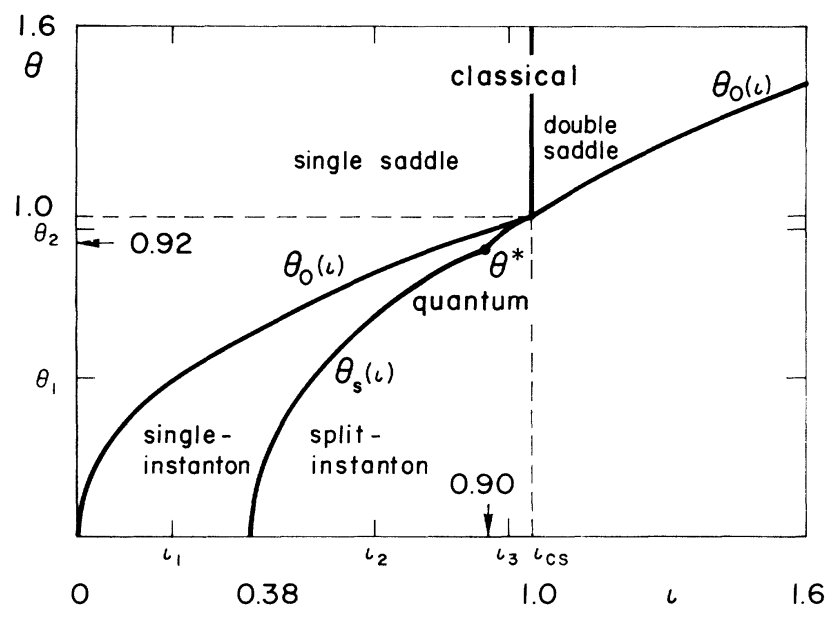

FIG. 4. The temperature-current phase diagram in terms of the dimensionless variables $\theta=(\eta \pi \beta) T / \hbar E_{J}$ and $\iota=\beta^{2}\left(2-I / I_{c}\right) / 4$ determining temperature $T$ and driving current $I$, respectively. At large currents $(\iota<1)$ the classical single-saddle regime at high temperatures is separated by the crossover line $\theta_{0}(\iota)=\sqrt{\iota}$ from the quantum single-instanton regime. This behavior is the same as for a system consisting of a single degree of freedom. For the present system consisting of two interacting degrees of freedom, several new critical lines arise in the temperature-current phase diagram. At high temperatures, the classical single-saddle regime is separated by the crossover line $\iota=1$ from the classical double-saddle regime; in the low-current region $(\iota>1)$ the double-saddle classical regime is separated from the two-instanton quantum one by the line $\theta_{0}(\iota)=(1+\sqrt{4 \iota-3}) / 2$; and in the large-current region $(\iota<1)$ a new critical line $\theta_{s}(\iota)=(\iota+\sqrt{\iota}-1)^{1 / 2}$ appears in the quantum regime, separating the single-instanton phase from the splitinstanton one. At low temperatures, the transition through the critical line $\theta_{s}(\iota)$ is smooth, analogous to a second-order phase transition. At temperatures near to the crossover temperature $\theta_{0}(\iota)$, the splitting of the instanton is abrupt, as in a first-order phase transition. $\theta^{*}$ is the tricritical point where the secondand first-order phase transitions merge. The decay rates along the lines $\iota_{i}=$ const $(i=1,2,3)$ and $\theta_{j}=$ const $(j=1,2)$ are illustrated in Figs. 5 and 6, respectively. tion (4) results in a lower action than that obtained with $q_{0}(z)=0$.

Using the Landau theory of phase transitions, ${ }^{17}$ we can treat the action as the thermodynamic potential of the system and we can determine the temperature-current phase diagram in terms of the dimensionless variables,

$$
\theta \equiv \frac{\eta \pi \beta}{\hbar E_{J}} T \text { and } \iota \equiv \frac{\varepsilon^{2} \beta^{2}}{4}=\frac{\left(2 I_{c}-I\right) \beta^{2}}{4 I_{c}}=\frac{1}{\alpha^{2}}
$$

The phase diagram comprises four phases (see Fig. 4). Let us consider initially the large currents region, $\iota<1$. At high temperatures, $\theta>\theta_{0}$, and very large currents (very small $\iota$ ) the system behaves classically and the decay process occurs through a single saddle. Crossing the boundary $\theta_{0}=\sqrt{\imath}$ (remember that $T_{0}=\hbar \varepsilon E_{J} / 2 \pi \eta$ ) we enter the quantum regime which is characterized by the single-instanton solution. Decreasing the current $I$ for a fixed temperature $T$, or decreasing the temperature for a fixed current, we finally cross the critical line $\theta_{s}^{2}=\iota+\sqrt{\iota}-1$ [see (18)] and reach the split-instanton phase. Instead, if the system is kept in the hightemperature regime and the current is reduced, a new classical phase arises at $\iota=1$ where the saddle splits into two (see also Fig. 3). Upon decreasing the temperature for a fixed value of current in the region $\iota>1$, the system enters the quantum regime characterized by the doubleinstanton solution. The crossover line $\theta_{0}$ separating the classical two-saddle regime from the quantum twoinstanton one, can be determined from the curvature of the potential at the saddle: from the equation of motion written in terms of Matsubara frequencies $\omega_{n}=2 \pi n T / \hbar$ we obtain

$$
\frac{2 \pi T_{0}}{\hbar} \eta=-\left.\frac{\partial^{2} V\left(p^{\prime}, q^{\prime}\right)}{\partial p^{\prime 2}}\right|_{s}, \quad \iota>1,
$$

where $\left.\left(\partial^{2} V / \partial p^{\prime 2}\right)\right|_{s}$ is the second derivative of the potential along the steepest descent of the saddle ( $p^{\prime}$ and $q^{\prime}$ are the two orthogonal main directions at the saddle). The crossover temperature $\theta_{0}(\iota)$ is then given by

$$
\theta_{0}(\iota)=\frac{1}{2}(1+\sqrt{4 \iota-3}) \text {. }
$$

Therefore, the point $\theta(\iota=1)=1$ is a crossing point between single and/or double and classical and/or quantum regimes. Although the two-instanton phase appears also for $\iota>1$, we are interested in the splitting of the instanton, which occurs only in the region $\iota<1$. In the following we will restrict our analysis to this region. The quantum tunneling rate for the two cases $\alpha>\alpha_{c}$ and $\alpha<\alpha_{c}$ will be discussed in Sec. IV.

\section{TUNNELING RATES AND PHASE DIAGRAM}

In Sec. III we have calculated the correction $\widetilde{q}^{(0)}(z)$ to $q_{0}(z)=0$, corresponding to the trajectory minimizing the action in the region $\alpha<\alpha_{c}$. Substituting Eq. (20) into Eq. (9), remembering that in the present approximation $\widetilde{p}=0$, and making use of $\hat{Q} q_{1}=2\left(\alpha-\alpha_{c}\right) q_{1}$, we obtain the quadratic form: 


$$
S=S_{0}+g\left(\alpha-\alpha_{c}\right) c_{1}^{2},
$$

where

$$
c_{1}^{2}=B^{2} \int_{-\pi}^{\pi} d z q_{1}^{2}(z)
$$

and $S_{0}$ is given by (8).

At this point, we have been able to solve the problem in the two regions $\alpha<\alpha_{c}$ and $\alpha>\alpha_{c}$. However, one has to remember that the constant $B$ in Eq. (20) is still unknown. Moreover, in the critical regime the eigenvalue $2\left(\alpha-\alpha_{c}\right)$ of the dangerous mode is small and it is no longer sufficient to consider only quadratic deviations from the classical trajectory. Both problems are solved by taking higher-order corrections into account, which is done in the following. From the Landau theory of phase transitions, with $c_{1}$ playing the role of the order parameter, we know that near the critical point where the coefficient of the quadratic term vanishes, the expansion of the free energy must contain also a fourth-order term. Hence, the action should take the form

$$
S=S_{0}+g\left(\alpha-\alpha_{c}\right) c_{1}^{2}+g \delta c_{1}^{4},
$$

where $\delta$ is a coefficient to be determined by considering higher-order corrections in the expansion of the action. In this case, the two eigenvalue problems for $\hat{P}$ and $\hat{Q}$ become coupled and we have to solve the inhomogeneous equations,

$$
\begin{aligned}
& \hat{P} \widetilde{p}=\frac{3}{2} \widetilde{q}^{2}, \\
& \hat{Q}\left(\alpha_{c}\right) \widetilde{q}=-2\left(\alpha-\alpha_{c}\right) \widetilde{q}+3 \widetilde{p} \widetilde{q},
\end{aligned}
$$

where the higher-order term $\propto \widetilde{p}^{2}$ in (25a) has been neglected [see (11a)]. The solution of the homogeneous equation for $\hat{Q}\left(\alpha_{c}\right)$ is $\widetilde{q}^{(0)}$. As $\alpha$ drops below its critical value $\alpha_{c}$, the solution $\widetilde{q}=\widetilde{q}^{(0)}$ is slightly distorted and we make the ansatz $\widetilde{q}=\widetilde{q}^{(0)}+\widetilde{q}^{(1)}$. Substituting this ansatz into (25b), neglecting $\widetilde{q}^{(1)}$ on the right-hand side, multiplying the entire equation by $\widetilde{q}^{(0)}$, and integrating we obtain

$$
3 \int_{-\pi}^{\pi} d z \tilde{p}(z) q_{1}^{2}(z)=2\left(\alpha-\alpha_{c}\right) \int_{-\pi}^{\pi} d z q_{1}^{2}(z),
$$

which is a useful expression relating the unknown function $\widetilde{p}(z)$ to the well-known dangerous mode $q_{1}(z)$. Inserting (25a), (25b), and (26) into (9) and neglecting the term proportional to $\widetilde{p}^{3}$, we eventually arrive at the following expression for the action,

$$
S=S_{0}+\frac{g}{2}\left(\alpha-\alpha_{c}\right) B^{2} \int_{-\pi}^{\pi} d z q_{1}^{2}(z) .
$$

Comparison with Eq. (24) minimized with respect to $c_{1}$,

$$
S=S_{0}-\frac{g\left(\alpha-\alpha_{c}\right)^{2}}{4 \delta}
$$

allows us to express the important parameter $\delta$ via the norm $u=\int_{-\pi}^{\pi} d z q_{1}^{2}(z)$ of the eigenfunction $q_{1}$ and the amplitude $B$,

$$
\delta=\frac{\alpha_{c}-\alpha}{2 u B^{2}}
$$

In a final step we have to determine the amplitude $B$. From (25a) we infer that $\widetilde{p}(z)=B^{2} R(z)$, where $R(z)$ is a function obeying the following inhomogeneous integral equation:

$$
\begin{array}{r}
{\left[2-3 p_{0}(z)\right] R(z)+\frac{T}{\pi T_{0}} \int_{-\pi}^{\pi} d z_{1} \frac{\partial R}{\partial z_{1}} \cot \left(\frac{z-z_{1}}{2}\right)} \\
=\frac{3}{2} q_{1}^{2}(z) .
\end{array}
$$

Using (26) we can easily verify that

$$
B^{2}=\frac{2 u}{3 v}\left(\alpha-\alpha_{c}\right),
$$

where $v=\int_{-\pi}^{\pi} d z q_{1}^{2}(z) R(z)$. Substituting now (31) into (29), we obtain the expression for the parameter $\delta$,

$$
\delta=-\frac{3 v}{4 u^{2}} .
$$

It remains to determine the function $R(z)$. Note that taking $\alpha=0$ in Eq. (5b), we obtain the homogeneous equation corresponding to (30). Following the same steps as we have done to find $q_{1}(z)$, we obtain an equation for the Fourier coefficients $\widetilde{R}_{n}$,

$$
(1+\gamma|n|) \widetilde{R}_{n}-2 \gamma \sum_{m=-\infty}^{\infty} \widetilde{R}_{m} e^{-b|n-m|}=\frac{3}{4} S_{n},
$$

where $\gamma \equiv T / T_{0}$ and

$$
\begin{aligned}
& S_{n}= \sum_{k=-\infty}^{\infty} C_{k} C_{n-k} \\
&=\sum_{k=-\infty}^{\infty}\left[\frac{\alpha_{c}}{\gamma}+|k|\right)\left(\frac{\alpha_{c}}{\gamma}+|n-k|\right) \\
& \\
& \times e^{-b(|k|+|n-k|)} .
\end{aligned}
$$

This equation can be solved analytically in two limits, namely, $T \approx T_{0}$ and $T \approx 0$. Near $T_{0}$, we have $\gamma \approx 1$ and $\alpha_{c} \approx 1$ [see (19)]. Considering only the three leading terms in the series (33) and (34) we obtain after some algebra $\delta=-(9 / 32 \pi)$, which characterizes a first-order phase transition since $\delta<0$. On the other hand, close to the absolute zero of the temperature, $T \approx 0, \gamma|n|$ can be treated as a continuous variable $x$. In this limit $b \approx \gamma$ [see (16)] and the equation to be solved takes the form

$$
\begin{aligned}
(1+|x|) \widetilde{R}(x)-2 \int_{-\infty}^{\infty} d y \widetilde{R}(y) e^{-|x-y|} & \\
= & \frac{3}{4} T \int_{-\infty}^{\infty} d \tau q_{1}(\tau)^{2} e^{-i 2 \pi T_{0} x \tau},
\end{aligned}
$$

where $\widetilde{R}(x)$ is a continuous function corresponding to the discrete Fourier coefficients $\widetilde{R}_{n}$. In this case, the value of $\delta$ turns out to be positive and thus characterizes a second-order phase transition. It follows that a temperature $T^{*}$ should exist where $\delta\left(T^{*}\right)=0 . T^{*}$ then corresponds to a tricritical point into which the curves for the first- and the second-order phase transitions merge. Below $T^{*}, \delta$ is positive and the transition is smooth if we vary $\alpha$ from $\alpha<\alpha_{c}$ to $\alpha>\alpha_{c}$, corresponding to a second- 
order phase transition. On the other hand, above $T^{*}$, the transition is abrupt, $\delta<0$, and it corresponds to a firstorder phase transition. From the result obtained for the underdamped case, ${ }^{11}$ where the phase diagram has the same features as pointed out here, we expect that the tricritical temperature $T^{*}$ must be very close to the crossover temperature $T_{0}$. Therefore, we can expand $\delta(\gamma)$ in a Taylor series around the value $\gamma=1$,

$$
\delta(\gamma)=\delta(1)+\left.\frac{\partial \delta}{\partial \gamma}\right|_{\gamma=1}(\gamma-1),
$$

and calculate the value of $\gamma$ for which $\delta(\gamma)=0$. Performing some tedious but simple calculations we obtain that the tricritical temperature $T^{*} \approx 0.97 T_{0}$.

We are now in a position to determine the behavior of the decay rate as a function of temperature and current in the classical and quantum regimes. As usual, the classical decay rate is given by the Arrhenius law,

$$
\Gamma_{t} \propto e^{-E(I) / T},
$$

where $T$ is the temperature and $E(I)$ is the activation energy, which is determined by the extremum of the free energy in (3) as a function of $(p, q)$. In the high-current region $(\iota<1)$ the activation energy $E_{s}(I)=4 E_{J} \varepsilon^{3}(I) / 3$, whereas in the low-current region $(\iota>1)$ the saddle splits and the activation energy becomes $E_{d}(I)=E_{J} \varepsilon^{3}(I)(2$ $\left.+3 \alpha-\alpha^{3}\right) / 3$. In the region $\iota<1$, as $T$ drops below the crossover temperature $T_{0}(I)$, the system enters the quantum regime and the decay rate takes the form

$$
\Gamma_{0} \propto e^{-S_{0}(I, T) / \hbar},
$$

where $S_{0}(I, T)=4 \pi \eta \varepsilon^{2}(I)\left[1-T^{2} / 3 T_{0}^{2}\right]$ is the action evaluated for the single-instanton solution. At $T=T_{0}$, the quantum and the classical results merge. For systems consisting of only one degree of freedom, the relaxation in the quantum regime is entirely described by Eq. (38). The saturation of the decay rate at low temperatures as obtained in (37) and (38) is in agreement with experimental observations on a rf SQUID. ${ }^{18,19}$ In our model containing two coupled degrees of freedom, the quantum regime is described by Eq. (38) only down to the temperature $T_{s}(I)$. Below $T_{s}(I)$ the instanton splits as a consequence of the development of a new unstable mode. For $\delta>0$, the decay rate can be obtained by singling out the integration over the dangerous mode,

$$
\begin{aligned}
\Gamma_{s} & \propto e^{-S_{0} / \hbar} \int_{-\infty}^{\infty} d c_{1} e^{-g\left[\left(\alpha-\alpha_{c}\right) c_{1}^{2}+\delta c_{1}^{4}\right] / \hbar} \\
& \simeq e^{-\left[S_{0}-g\left(\alpha-\alpha_{c}\right)^{2} / 4 \delta\right] / \hbar}
\end{aligned}
$$

where we have made use of (28) in the last expression. Comparison of the results (38) and (39) shows that $\Gamma_{s}>\Gamma_{0}$ and thus the instanton splitting leads to a relative increase in the decay rate. For negative $\delta$, a term proportional to $c_{1}^{6}$ has to be considered in the action, i.e., the term proportional to $\widetilde{p}^{3}$ should be retained in Eq. (9) in order to provide a complete description.

Defining the new dimensionless variable for the loga- rithm of the decay rate $\Gamma$,

$$
\sigma \equiv-\frac{3 \beta^{2} \hbar}{16 \pi \eta} \ln \Gamma
$$

and using the variables defined in (21), $\theta$ (related to the temperature $T$ ) and $\iota$ (related to the difference between the bias current $I$ and the critical current $2 I_{c}$ in the system) one can rewrite the decay rates in the simple form (remember $\iota<1$ ),

$$
\begin{aligned}
\sigma_{t} & =\frac{2 \iota^{3 / 2}}{\theta}, \\
\sigma_{0} & =3 \iota-\theta^{2}, \\
\sigma_{s} & =3 \iota-\theta^{2}-\kappa^{2}(\iota, \theta),
\end{aligned}
$$

where $\kappa^{2}(\iota, \theta)=3 \beta^{2} \hbar g\left(\alpha-\alpha_{c}\right)^{2} / 64 \pi \eta \delta$. Note that $\alpha$ is a function of $\iota$, and $\alpha_{c}$ and $\delta$ are functions of both $\iota$ and $\theta$.

Let us analyze the result (41) in detail (Figs. 4-6). To begin with, we keep the current $(\iota)$ fixed and discuss the functional dependence $\sigma(\theta)$, see Fig. 5. For a large

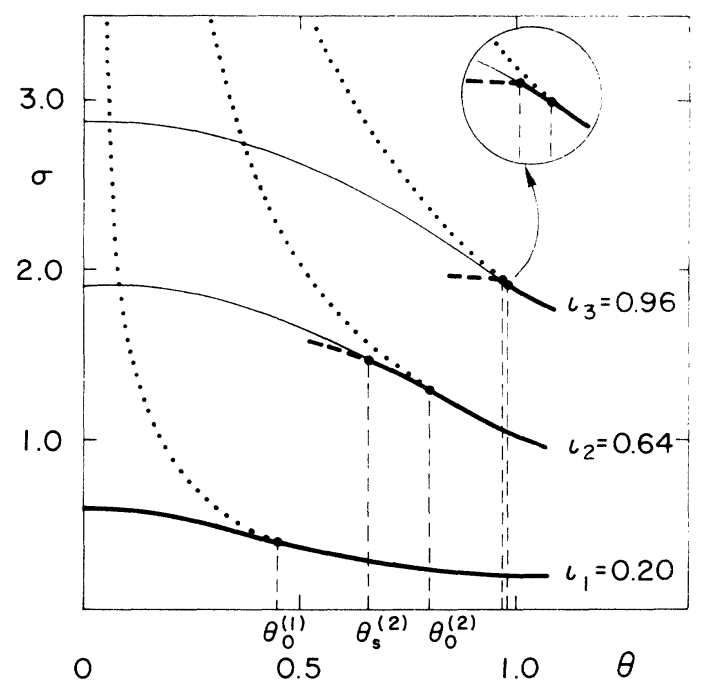

FIG. 5. Logarithm of the decay rate vs temperature in terms of the dimensionless variables $\sigma=-\left(3 \beta^{2} \hbar / 16 \pi \eta\right) \ln \Gamma$ and $\theta=(\eta \pi \beta) T / \hbar E_{J}$ for three different regions within the phenomenological temperature-current phase diagram (see Fig. 4). For a large current $\left(\iota_{1}=0.20\right)$ and at high temperatures, the system decays following Eq. (41a), $\sigma_{t} \propto 1 / \theta$ (dotted line). Upon reducing the temperature, the quantum regime is reached at $\theta_{0}^{(1)}=0.45$ and the decay rate becomes $\sigma_{0} \propto$ const $-\theta^{2}$ [see (41b)]. The behavior is the same as for a system consisting of a single degree of freedom because the coupling term between the two phases in the SQUID is relevant and forces them to decay symmetrically. If we fix the bias current to a lower value $\left(\iota_{2}=0.64\right)$ the system decays by thermal activation at high temperatures, enters the quantum regime described by Eq. (41b) at $\theta_{0}^{(2)}=0.80$, and then reaches a new phase in the quantum regime at $\theta_{s}^{(2)}=0.66$, where the instanton splits and the relative value of the decay rate increases [dashed line, see (41c)]. The transition at $\theta_{s}^{(2)}$ is smooth, like in a second-order phase transition. Reducing further the bias current $\left(\iota_{3}=0.96\right)$, the system behaves in the same manner $\left(\theta_{0}^{(3)}=0.98\right.$ and $\left.\theta_{s}^{(3)}=0.97\right)$ except for an abrupt change in the action at $\theta_{s}^{(3)}$, a behavior corresponding to a first-order phase transition. 


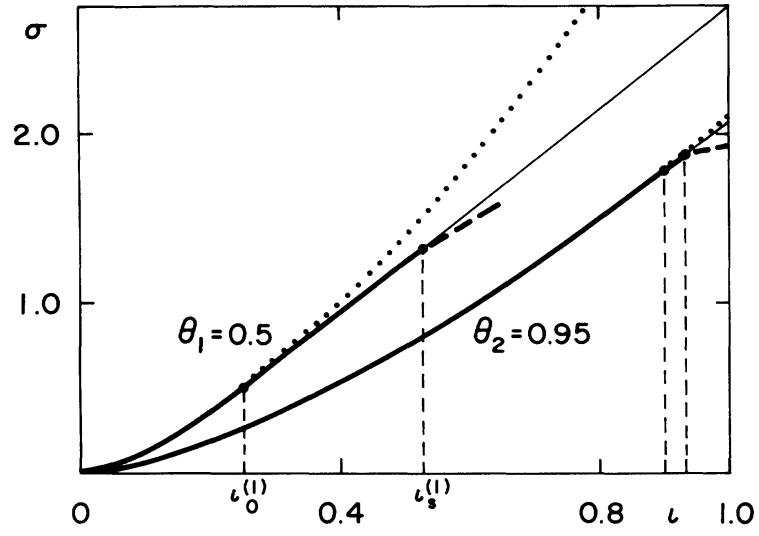

FIG. 6. Logarithm of the decay rate vs current in terms of the dimensionless variables $\sigma=-\left(3 \beta^{2} \hbar / 16 \pi \eta\right) \ln \Gamma$ and $\iota=\beta^{2}\left(2-I / I_{c}\right) / 4$ for fixed values of temperature $\left(\theta_{1}=0.5, \theta_{2}=0.95\right)$. For large currents (small $\left.\iota\right)$, the system is in the thermal regime described by Eq. (41a), $\sigma_{t} \propto \iota^{3 / 2}$ (dotted line). At the value $\iota_{0}^{(1)}=0.25\left(\iota_{0}^{(2)}=0.90\right)$, the single-instanton regime is reached [see Eq. (41b), $\sigma_{0} \propto 3 \iota$-const] and at $\iota_{s}^{(1)}=0.53\left(\iota_{s}^{(2)}=0.93\right)$, the instanton splits [dashed line, see Eq. (41c)]. The splitting can be smooth (case 1) or sharp (case 2).

current $\left(\iota_{1}=0.20\right.$, remember that large currents correspond to small $\iota$ ) and at high temperatures, the system decays following (41a), $\sigma_{t} \propto 1 / \theta$. Upon reducing the temperature, the system enters the quantum regime at $\theta_{0}^{(1)}=0.45$ and relaxes according to $(41 \mathrm{~b}), \sigma_{0} \sim$ const $-\theta^{2}$. At large bias current, the coupling between the two phases in the SQUID is very effective and they decay symmetrically. Reducing the bias current $\left(\iota_{2}=0.64\right)$, the system decays by thermal activation at high temperatures, enters the quantum regime described by the single instanton at $\theta_{0}^{(2)}=0.8$, and then reaches a new phase in the quantum regime at $\theta_{s}^{(2)}=0.66$ (see also Fig. 4), where the instanton splits and the decay rate $\Gamma_{s}$ is larger than the value $\Gamma_{0}$ obtained for the single-instanton solution [see Eq. (41c)]. The transition at $\theta_{s}^{(2)}$ is smooth, like in the second-order phase transition. If the bias current is further lowered $\left(\iota_{3}=0.96\right)$, the system behaves in the same manner except for an abrupt change in the action (corresponding to a first-order transition) as the splitinstanton region is entered. The behavior of the system at fixed temperature $\theta$ and with varying current $\iota$ is illustrated in Fig. 6. The second-order transition to the splitinstanton region is probably difficult to observe experimentally. However, the discontinuity in the decay rate occurring for $\iota_{3}=0.96$ in Fig. 5 and for $\theta_{2}=0.95$ in Fig. 6 is a signal of the onset of instanton splitting.

\section{CONCLUSIONS}

In the present work, the quantum decay of a currentcarrying state in a dc SQUID has been studied in the overdamped limit. The analysis of this system comprising two coupled macroscopic quantum degrees of freedom has shown that a much richer behavior is developed than in a system with only a single degree of freedom.
Beyond the crossover temperature $\theta_{0}(\iota)$, which exists also in the latter case and indicates the appearance of a nontrivial trajectory in imaginary time, an additional critical temperature $\theta_{s}(\iota)$ arises in this system marking a qualitative change in the nature of the tunneling process.

Considering the problem in terms of the Landau theory of phase transitions, a phase diagram relating the temperature $T$ and the bias current $I$ has been constructed. Four distinct phases have been found, depending on the values of the external parameters (temperature and bias current) which influence the shape of the potential. At high temperatures, the decay out of the metastable state is due to thermal activation and the system behaves classically. In this regime the decay rate is given by the usual Arrhenius law. Depending on the value of the external current, two different situations can be realized in the classical limit. At high temperatures $\theta(\iota)>\theta_{0}(\iota)$ and large currents $\iota<1$, the system escapes through a single saddle, whereas at lower currents $(\iota>1)$ the saddle splits into two. For $\iota<1$, upon decreasing the temperature, in the region $\theta(\iota)<\theta_{0}(\iota)$, the system decays due to quantum tunneling through the barrier. The quantum regime is characterized by two different regions. At large enough bias current $I$ (small $\iota$ ) and high enough temperature $T$, i.e., in the region $\theta_{s}(\iota)<\theta(\iota)<\theta_{0}(\iota)$, the phases $\varphi_{1}$ and $\varphi_{2}$ in the two Josephson junctions are strongly correlated and they decay symmetrically. This behavior is the same as for a system composed of only a single degree of freedom coupled to the environment. Its dynamics is described by the single-instanton solution and has been discussed before by Larkin and Ovchinnikov ${ }^{3}$ for the case of strong dissipation. Below the critical line $\theta_{s}(\iota)$, the instanton splits and two degenerate extremal trajectories appear. In this regime the two phases are only weakly correlated and they decay in an asymmetric way. We have calculated analytically the split-instanton solution corresponding to the quantum behavior in the region $\theta(\iota)<\theta_{s}(\iota)$. Surprisingly, the solution for the overdamped case has a very simple form, in contrast to the elliptic functions obtained for the underdamped case. ${ }^{11}$ The splitting of the instanton reduces significantly the decreasing of the tunneling rate in the quantum regime. Focusing our attention on this new transition line $\theta_{s}(\iota)$, we have observed that near the critical regime, the fluctuations of the mode corresponding to a transition between the two trajectories become large, requiring to go beyond the quadratic approximation in the action (the fourth-order term is needed in this case). At low temperatures, the transition through the critical region is smooth, analogous to a second-order phase transition. Instead, at temperatures near to the crossover temperature $\theta_{0}(\iota)$ separating the quantum regime from the classical one, the splitting of the instanton is abrupt, as in a first order-phase transition. Above and close to the tricritical point $\theta^{*}$, where the second- and first-order phase transitions merge, the dangerous mode fluctuates so strongly that sixth-order corrections have to be included in the action.

It would be interesting to observe experimentally the variation of the decay rate as a function of the temperature $T$ and the bias current $I$ in the region corresponding 
to the instanton splitting. In Ref. 20, the transition rate from the zero-voltage metastable minimum in the washboard potential of a dc SQUID has been measured as a function of applied flux and temperature. However, the devices used in this study are characterized by very small inductances $(\beta<<1)$ and in this limit, the splitting cannot be observed. The inductance $L$ of the loop is given by the diameter of the device, which in turn also determines the noise level. As a consequence, experimental verification of the instanton-splitting phenomenon requires an optimized device with a large enough inductance to make the effect observable but still small enough to avoid the problem of noise. Recently, the behavior of a dc SQUID in the classical regime corresponding to the doubling of the saddle in the bidimensional surface potential has been experimentally observed. ${ }^{21}$ However, in the quantum regime, experiments remain to be performed in order to observe the dynamical splitting of the instanton.

\section{ACKNOWLEDGMENTS}

We thank A. O. Caldeira, P. Hänggi, and B. Kramer for fruitful discussions. B. I. acknowledges financial support from the Swiss National Foundation. One of us (C. M. S.) was supported by Conselho Nacional de Desenvolvimento Científico e Tecnológico under Grant No. 201360/92-6 (NV).
${ }^{1}$ A. O. Caldeira and A. J. Leggett, Phys. Rev. Lett. 46, 211 (1981).

${ }^{2}$ A. O. Caldeira and A. J. Leggett, Ann. Phys. (N.Y.) 149, 374 (1983).

${ }^{3}$ A. I. Larkin and Yu. N. Ovchinnikov, Pis'ma Zh. Eksp. Teor. Fiz. 37, 322 (1983) [JETP Lett. 37, 382 (1983)].

${ }^{4}$ H. Grabert, U. Weiss, and P. Hänggi, Phys. Rev. Lett. 52, 2193 (1984).

${ }^{5}$ H. Grabert and U. Weiss, Z. Phys. B 56, 171 (1984).

${ }^{6}$ S. Coleman, Phys. Rev. D 15, 2929 (1977).

${ }^{7}$ C. Callan and S. Coleman, Phys. Rev. B 16, 1762 (1977).

${ }^{8}$ I. Affleck, Phys. Rev. Lett. 46, 388 (1981).

${ }^{9}$ A. Barone and G. Paterno, Physics and Applications of the Josephson Effect (Wiley, New York, 1982).

${ }^{10}$ Yong-Cong Chen, J. Low. Temp. Phys. 65, 133 (1986).

${ }^{11}$ B. I. Ivlev and Yu. N. Ovchinnikov, Zh. Eksp. Teor. Fiz. 93, 668 (1987) [Sov. Phys. JETP 66, 378 (1987)].

${ }^{12}$ P. Hänggi, P. Talkner, and M. Borkovec, Rev. Mod. Phys. 62, 251 (1990).

${ }^{13} \mathrm{U}$. Weiss, in Quantum Dissipative Systems, Series in Modern
Condensed Matter Physics, edited by J. E. Dzyaloshinskii, S. O. Lundqvist, and Yu Lu (World Scientific, Singapore, 1993), Vol. 2.

${ }^{14}$ G. Blatter, V. Geshkenbein, and V. Vinokur, Phys. Rev. Lett. 66, 3297 (1991).

${ }^{15}$ B. Ivlev, Yu. N. Ovchinnikov, and R. S. Thompson, Phys. Rev. B 44, 7023 (1991).

${ }^{16}$ I. S. Gradshteyn and I. M. Ryzhik, Table of Integrals, Series and Products (Academic, New York, 1980).

${ }^{17}$ L. D. Landau and E. M. Lifshitz, Statistical Physics (Pergamon, New York, 1958).

18 J. M. Martinis, M. H. Devoret, and J. Clarke, Phys. Rev. Lett. 55, 1543 (1985).

${ }^{19}$ M. H. Devoret, J. M. Martinis, and J. Clarke, Phys. Rev. Lett. 55, 1908 (1985).

${ }^{20}$ F. Sharifi, J. L. Gavilano, and D. J. Van Harlingen, Phys. Rev. Lett. 61, 742 (1988).

${ }^{21}$ V. Lefreve-Seguin, E. Turlot, C. Urbina, D. Esteve, and M. H. Devoret, Phys. Rev. B 46, 5507 (1992). 